\title{
Maine Medical Center Cancer Institute's Prostate Cancer Clinic
}

\begin{abstract}
A virtual clinic in Portland, Maine, ensures patients have uniform access to all specialties By Moritz Hansen, MD
\end{abstract}

At Maine Medical Center Cancer Institute in Portland,

Maine, prostate cancer represents one of our largest

volumes of cancer patients. In 2007 we set out to

develop a virtual prostate cancer clinic. At the outset,

we solicited provider and patient feedback and set a goal

to develop a care approach that would be patient and

family focused (see Figure 1, page 27). The foundation of

our multidisciplinary prostate cancer clinic was based

on a commitment to improving the care and services

offered to our prostate cancer patients and their families. Our goal was to ensure that all prostate cancer patients had uniform access to the consultations they would need. We also wanted to distinguish our program by incorporating a decision-making process that would improve the quality of our care. The implementation plan was threefold: 1) develop a uniform process for bringing prostate cancer patients into our healthcare system, 2) record clinical outcomes, and 3) use this data as a benchmark to compare our prostate cancer clinic to other nationally prominent programs. he first step to developing a virtual prostate cancer clinic was to bring all the players to the table. Fortunately, in our community we have a limited number of key players involved in prostate cancer care. Maine Medical Partners-Urology (a multispecialty group practice affiliated with Maine Medical Center) and Spectrum Medical Group (a private radiation oncology practice) partner with Maine Medical Center's Cancer Institute to treat prostate cancer patients. Other practices involved in providing patient care at our Cancer Institute, such as the Maine Center for Cancer Medicine, a private medical oncology practice, were included in the planning process. While medical oncologists often do not see prostate cancer patients unless other treatment modalities have failed, the medical oncologists were eager to engage with patients earlier in the disease process. For other community cancer centers, perhaps with more players involved, developing a model prostate cancer clinic may be more challenging.

\section{Our Prostate Cancer Workgroup}

In 2007 Maine Medical Center Cancer Institute established a Prostate Cancer Workgroup. The group's task was to create a clinic model that would allow patients to be seen by all specialties involved in the management of prostate cancer. In prostate cancer, however, there is not always uniformity surrounding treatment decisions. To resolve this issue, the group had to reach consensus on how key providers could collaborate in a way that would benefit prostate cancer patients.

An active surveillance protocol was the first area in which our urologists, radiation oncologists, and medical oncologists were able to come to a consensus. The Prostate Cancer Workgroup developed a protocol that outlined how these patients would be followed and what mechanisms would trigger treatment (see page 30). Starting with this patient subset, the workgroup was then able to adopt a best practice treatment guideline, using published guidelines for reference. ${ }^{1}$ Mechanisms were put in place to ensure that these patients are seen at regular intervals. This consensus was an important milestone for our virtual prostate cancer clinic and one that required a high degree of cooperation among all participants.

Consensus was soon reached in another key area-the educational information that would be distributed to all prostate cancer patients. The Prostate Cancer Workgroup developed a packet of information describing all of the surgical and radiation treatment options and follow-up care. No matter where patients enter the healthcare system-at the urology practice, at the radiation oncology practice, or at the hospital-they receive the same packet of information.

\section{Our Multidisciplinary Prostate Tumor Board}

Prior to the development of the Prostate Cancer Workgroup, interaction between the urology and radiation oncology practices was limited. Establishing communication forums, such as a multidisciplinary prostate tumor board attended by representatives from all practices, and hiring a dedicated clinical patient navigator employed by the hospital were key to greatly improving our interpractice communication.

Today, Maine Medical Center Cancer Institute's Genitourinary Cancer Program uses a multidisciplinary forum to discuss genitourinary cancer patient clinical information, as well quality improvement measures designed to improve care for all prostate cancer patients.

Our genitourinary tumor conference meets on the first 


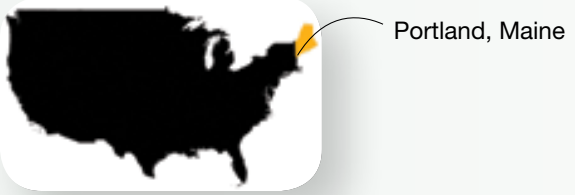

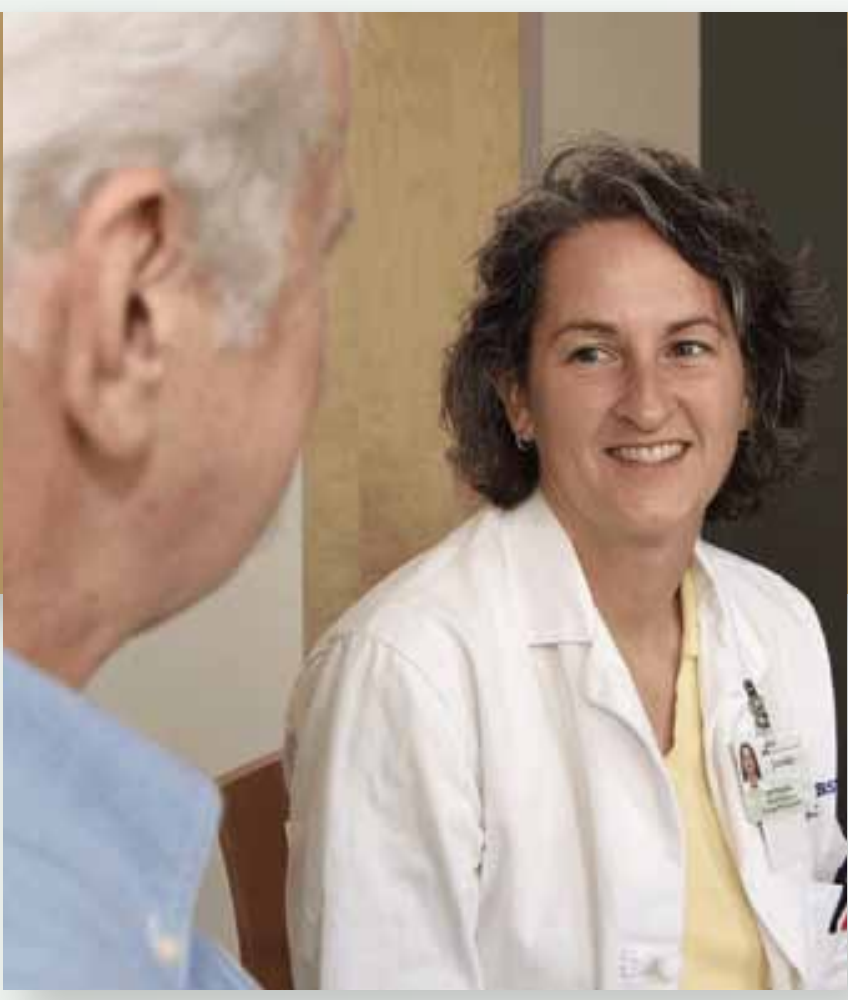

Jennifer Powers, MS, BSN, RN, CCRC, clinical patient navigator for Genitourinary Cancer at Maine Medical Center Cancer Institute, Portland, Maine.

and third Thursday of each month. Cases are submitted in advance to the Genitourinary Clinical Patient Navigator. (For more on this key position, see the following page.) All genitourinary cancer cases are welcome and are accepted on a first-come, first-served basis. The multidisciplinary team, which includes medical oncology, pathology, radiation oncology, urology, and the clinical patient navigator, reviews each case.

Additionally, our virtual prostate cancer clinic has interdepartmental support from radiology, pathology, and the tumor registry. While physician attendance is voluntary, our expectation is that this multidisciplinary approach to care will ultimately help us remain focused on a care approach that supports informed decision making and is patient and family centered.

Prostate cancer patients may have multiple-often equally effective-management options. Often, prostate cancer does not require an urgent treatment as with other cancers. And in some cases, the best management approach is active surveillance or a watchful waiting approach. For

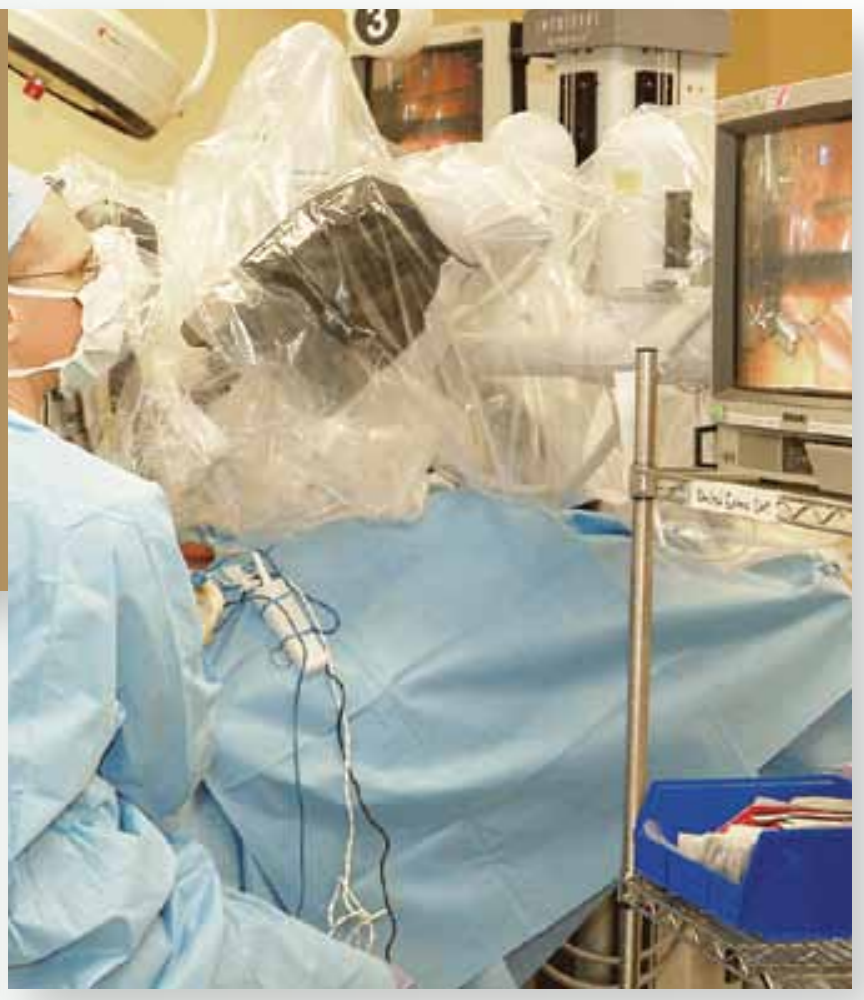

Moritz Hansen, MD, medical director, Genitourinary Cancer Program, Maine Medical Center Cancer Institute, Portland, Maine, performs surgery using the da Vinci Robotic Surgical System.

these and other reasons, attending physicians are not always in complete agreement about treatment decisions. To help in these situations, our tumor board uses treatment algorithms that have been jointly developed by our multidisciplinary team.

\section{Our Clinical Patient Navigator}

Another key component of our virtual prostate cancer clinic was the creation of a full-time position to help patients navigate the decision-making process and to organize the genitourinary tumor board meetings. In our model, a hospitalemployed cancer clinical patient navigator is responsible for bringing physicians from all relevant private practices together to meet on a regular basis. This neutral, thirdparty healthcare professional educates patients about all management options for prostate cancer and ensures that every patient is seen by the appropriate surgical, radiation, or medical oncology specialist.

In place since April 2008, our clinical patient navigator 
focuses on the overall picture of the prostate cancer patient and understands how the patient and his family are affected by treatment. The navigator assists patients and families as challenges arise and helps anticipate issues going forward. Depending on the acuity of patients, our clinical patient navigator supports between 200-250 patients each year. Job responsibilities include:

- Empowering prostate cancer patients and their families

- Advocating for prostate cancer patients and their families

- Supporting physicians

- Ensuring that prostate cancer patients have the necessary information to make a fully informed treatment decision and access to all available resources

- Encouraging prostate cancer patients to meet with all specialties-urology, radiation oncology, and medical oncology

- Referring patients and families to social services and nutritional and genetic counseling.

One specific area that our clinical patient navigator has focused on is cancer treatment regret-making a decision without knowing all of the treatment options. Fortunately, our virtual prostate cancer clinic has been able to mitigate this experience

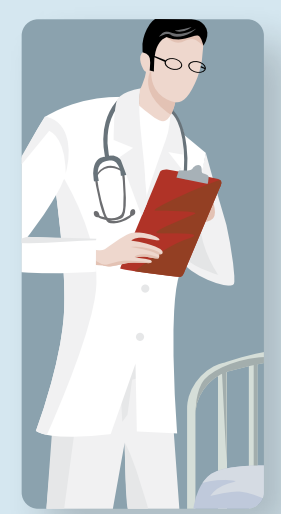
for patients.

Our clinical patient navigator also serves as the frontline mechanism for screening patients for appropriate clinical trials offered at the medical oncology practice, the radiation oncology practice, or the urology practice. Medical oncologists are able to counsel high-risk patients regarding ongoing clinical chemotherapeutic trials. Prostate patients who wish to have definitive treatment, but who are undecided between radiation and surgery, can also consult with a medical oncologist.

In addition to the clinical patient navigator, other members of the cancer support service team meet with prostate cancer patients and family members, including a dedicated outpatient oncology social worker, a certified oncology nutritionist, and an American Cancer Society (ACS) patient navigator. The ACS patient navigator is available to all of our cancer patients and families that need assistance with issues related to finances, insurance, transportation, and more. The ACS navigator supports between 500-750 patients per year.

\section{Our Core Services}

Our virtual prostate cancer clinic offers a full range of management options, to include:

- Active surveillance

- Robotic-assisted prostatectomy

- Open radical prostatectomy

- High-dose rate brachytherapy

- Low-dose rate brachytherapy (prostate seed implants)

- External beam radiation therapy (IMRT)

- Cryosurgery

- Hormonal therapy

- Chemotherapy

- Clinical trials.
While having cutting-edge technology, such as a robotic surgical program, can increase referrals to a prostate cancer clinic, not all community cancer centers need to offer a full range of services. Instead, consider establishing relationships with larger cancer centers that do offer certain treatments. The key to this cooperative relationship is to ensure that patients are returned to their community for follow-up care after treatment.

\section{Quality Care Indicators}

Maine Medical Center Cancer Institute has been prospectively collecting outcomes data on prostate cancer since 1998. Our Prostate Cancer Database is a dynamic measurement tool that uses a numerical graduation to collect quality of life indicators beyond the standard cancer registry data set, including information on pre- and post-treatment quality-of-life parameters, such as urinary, bowel, and sexual dysfunction (see Figure 2, page 32). The database includes nearly 2,000 patients and consists of demographics, treatment-specific complication rates, and quality-of-life outcomes. Patients managed with surgery, radiation therapy, and active surveillance are followed in the same manner.

Nurses and physicians collect these data from the urology and radiation oncology practices at each patient visit. (Radiation oncology services are provided at the hospital and affiliated off-site locations; the urology practice has its own offsite location.) The complexity of data interpretation, informed decision making, and individualized treatment requires the coordination of the clinical patient navigator. Clinical outcome data are stratified by age, management option, and follow-up interval. The data are then integrated into the workflow of the hospital's tumor registry. At the Prostate Workgroup's quarterly meeting, outcome data is reviewed and then reported annually to Maine Medical Center Cancer Institute's Steering Committee.

Initially, we were challenged to get physicians motivated to participate and believe in the benefits of collecting and maintaining clinical outcomes in a standardized manner. Some physicians were worried about sharing outcomes data-particularly if their outcomes data were not as positive as other physicians. We were able to work through this issue by assuring physicians of anonymity. Our data are an aggregate of all physicians; no one physician can be singled out. The only individual who has access to surgery data stratified by physician is the medical director of the Genitourinary Cancer Program, who reviews this information annually for quality improvement initiatives.

Outcomes data for the prostate cancer clinic are housed at the Maine Medical Center Cancer Institute and disseminated in multiple ways, including:

- Maine Medical Center Cancer Institute Physician Symposia

- Maine Medical Center's Annual Community Forum

- Various local groups, such as prostate cancer support groups

- Prostate cancer patients and family members. 


\section{Figure 1. Prostate Cancer Clinic Navigator Pathway}

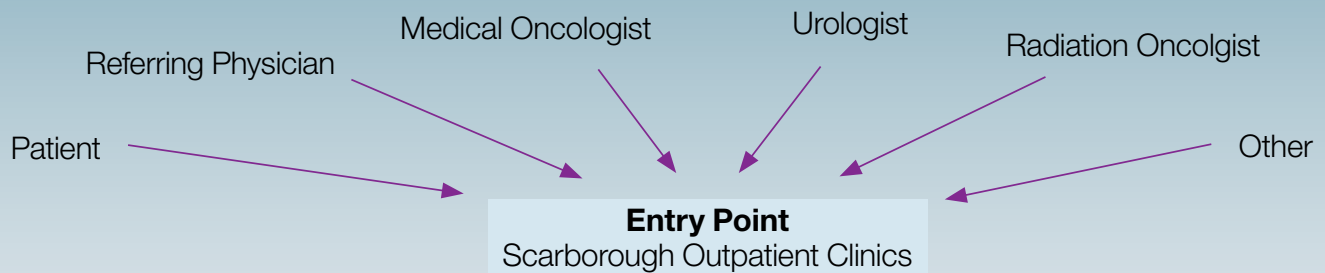

\section{Support Services:}

(Barriers to timely diagnosis and treatment)

Education

- Health insurance and/or financial concerns

- Transportation

- Physical and/or psychosocial needs

- Communication and/or cultural needs

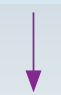

Navigator

(Triage)

Follow-up

Post treatment

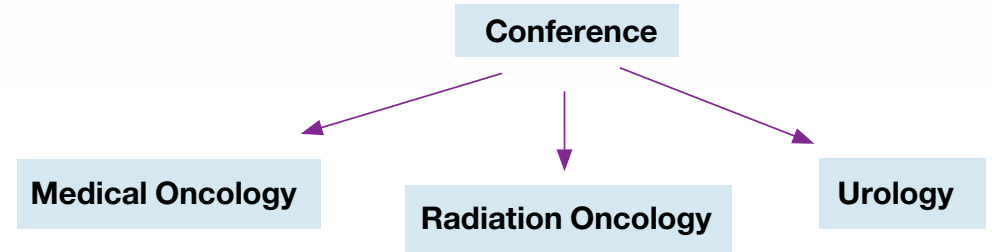

Community prostate cancer referral centers have an obligation not only to record the care that is provided to patients, but also to communicate this information back to referring physicians. We used our outcomes data in our 2009 Prostate Cancer Quality Report on Clinical Outcomes, a comprehensive guide that is mailed to all referring physicians in Maine. The purpose of the guide is to provide detailed information to our referring community about the depth and breadth of services available at Maine Medical Center and to help facilitate collaboration between specialtiesultimately improving patient care.

\section{Patient Volume and Market Share}

Maine Medical Center Cancer Institute monitors marketshare data from the top five cancer sites, including prostate cancer. Our Oncology Information Services tracks patient volume, incidence, and market share. Volume is tracked through:

- The hospital's tumor registry (including prostate cancer patients on active surveillance)

- The hospital's radiation oncology services

- Services offered at Maine Medical Partners-Urology (a multi-specialty group subsidiary affiliated with Maine Medical Center, which provides patient-centered care).

The use and distribution of volume and market-share data has evolved over the years. For example, our physicians now have access to three-year or five-year averages when making programmatic decisions. We also keep track of the number of prostate cancer patients who choose surgery and those who choose radiation treatment. We are careful not to over simplify how we interpret the data and take into account other information such as the age of the patient and co-morbid medical conditions.

\section{Making a Business Plan}

At Maine Medical Center, the prostate cancer clinic is one part of the overall budget for the Cancer Institute. The clinical patient navigator, nutritionist, and social worker are budgeted hospital positions. The ACS navigator is a partnership with the hospital and the American Cancer Society.

Our hospital does not use a return on investment model to make its funding decisions. Instead decisions about new equipment and additional staff are based on needs and 
merit-what is best for our patients. Maine is also a Certificate of Need state, so we are regulated in what we can do in our budget process.

We have encountered a fair amount of resistance from payers regarding the more costly treatment options, such as robotic surgery. Our Prostate Cancer Database shows that while some procedures are more expensive, they are also associated with decreased hospital readmissions and treatment complications. Having access to quality outcomes data that can be shared with payers has helped in contract negotiations.

When purchasing and/or instituting new technologies, we have also used our Prostate Cancer Database to compare clinical outcomes to existing treatments.

\section{Marketing Our Virtual Prostate Cancer Clinic}

Maine Medical Center Cancer Institute recently undertook a communications and marketing planning process that included prostate cancer as a priority area in its community awareness efforts. While target audiences will shift and vary dependent upon the exact service or product being promoted, we will target the following internal and external stakeholders to receive key messages and marketing information about our virtual prostate cancer clinic.

\section{Internal stakeholders \\ - Board of Trustees \\ - Executive leadership \\ - Physicians (specifically, medical staff leaders) \\ - Management \\ - Employees \\ - Volunteers.}

\section{External Stakebolders}

- Consumers (potential patients)

- Physicians-both primary care physicians (referral sources) and specialists

- Media

- Employers

- Civic organizations

- Business and opinion leaders.

As noted previously, Maine Medical Center Cancer Institute is the first hospital in the region to publish an outcomes data and referral guide. The guide provides an overview of Maine Medical Center Cancer Institute, an in-depth look at our clinical outcomes, and a review of program-related people and services that make it all possible. The guide was recently mailed to all primary care physicians and select specialists in Maine and also made available on our website at: www.mmc.org/CancerInstituteOutcomes. Our other marketing efforts include:

- Developing TV, radio, and print ads

- Enhancing our website by including patient and physician testimonials about the prostate cancer clinic

- Developing a capabilities brochure for referring physi- cians that describes the major attributes of the prostate cancer clinic

- Scheduling annual symposia for physicians and local civic groups

- Arranging for physicians from the prostate cancer clinic to speak at various dinners and other events

- Writing formal thank-you letters to referring physicians

- Developing patient brochures describing prostate cancer clinic services.

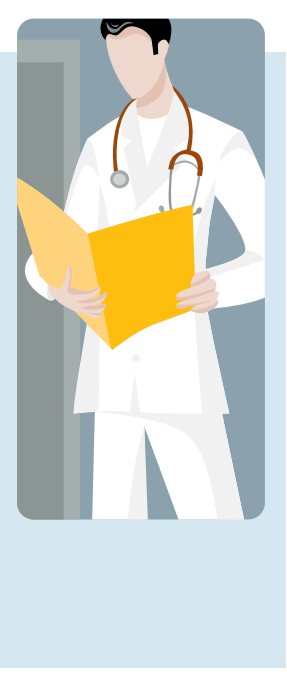

\section{Outreach Efforts}

Annually, Maine Medical Center Cancer Institute offers a free prostate cancer screening to men without routine access to medical care. This effort is supported by the hospital and staffed by hospital-employed and private practice physicians. We screen between 100-185 men. Everyone is seen by a physician and has a PSA test and rectal examination. Patients are then absorbed into the healthcare system. So far, the cost of this outreach effort has not been a major issue for the hospital. Maine Medical Center Cancer Institute has a very generous uncompensated care plan. Everyone with cancer is treated-regardless of their ability to pay.

We work collaboratively with Maine prostate cancer survivor and advocacy groups. A brochure listing contact information for these groups is available at most sites providing cancer care throughout the state.

We also conduct focused physician education efforts. In 2007 community dialogue focused on prostate screening and treatment. Physician education strategies included developing Web content, providing physicians from our virtual prostate cancer clinic for grand rounds and other symposia presentations around the state, and participating in the development of state-wide prostate cancer screening guidelines. OI

Moritz Hansen, MD, is medical director, Genitourinary Cancer Program, Maine Medical Center Cancer Institute, Portland, Maine.

The author would like to thank the following people who contributed to this article: Barbara Grillo, director, MMC Cancer Institute; Donna Green, RN, BSN, $\mathrm{BA}, \mathrm{OCN}$, clinical nurse manager at the MMC Cancer Institute; Melanie Feinberg, oncology information services manager at the MMC Cancer Institute; and Leslie Weeks, RTT, MSB, business manager at the MMC Cancer Institute, Portland, Maine.

\section{Reference}

${ }^{1}$ Klotz L. Active surveillance for prostate cancer: for whom? J Clin Oncol. 2005;23(32):8165-8169. 
Maine Medical Center

Cancer Institute

centered around you

\section{Genitourinary Navigator Referrals}

\section{Urology, Radiation Oncology, or Medical Oncology (currently) \\ Referrals come via fax, email, and or verbal communication.}

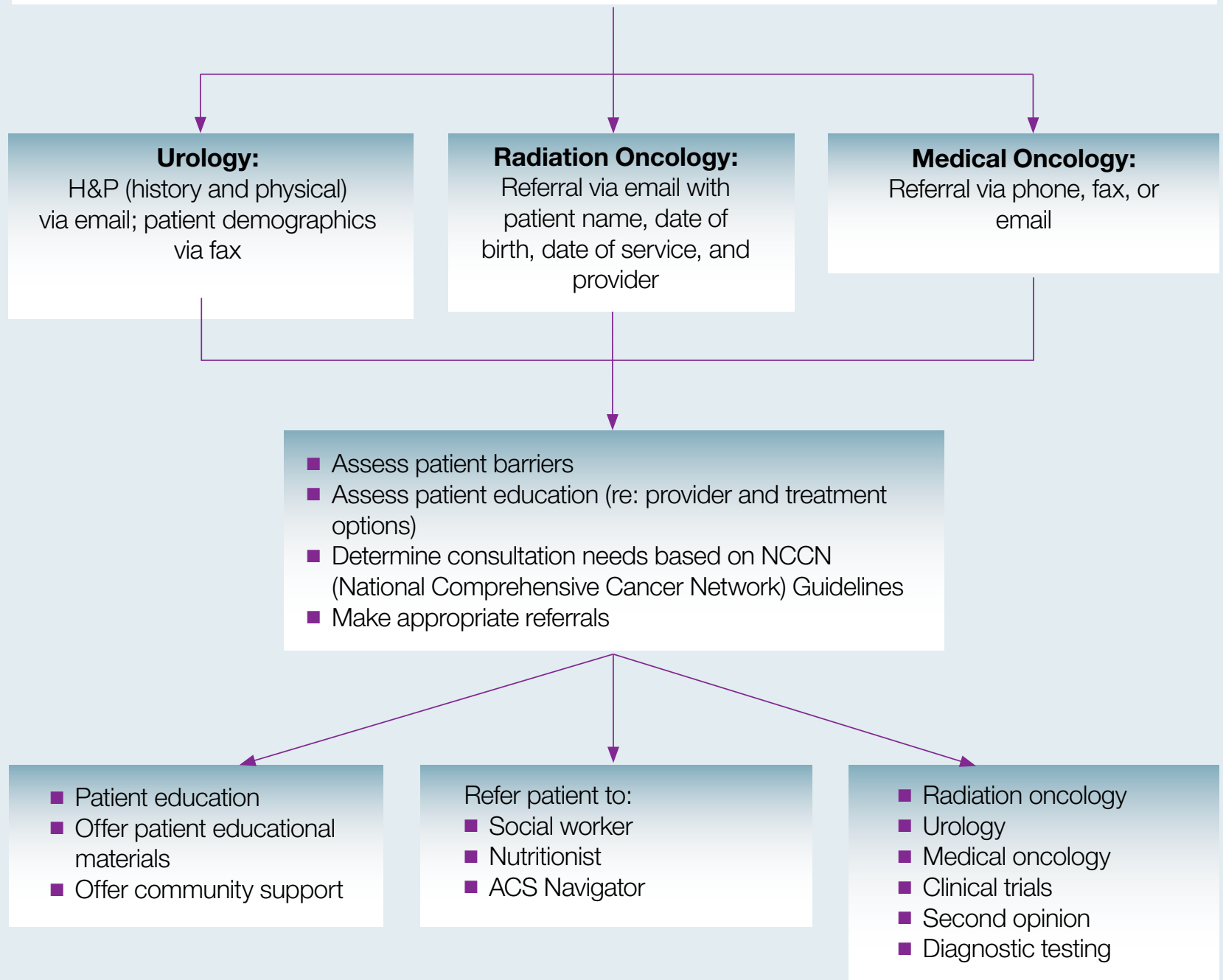

\footnotetext{
Appropriate GU Navigator Referrals include:

- All newly diagnosed prostate cancer patients

- Recurrent prostate cancer patients

- Newly diagnosed or recurrent renal cancer patients

- Newly diagnosed or recurrent bladder cancer patients

- Newly diagnosed or recurrent testicular cancer patients

- Newly diagnosed or recurrent penile cancer patients

- GU tumor conference patients
}

Questions? Please call (insert appropriate name, credentials, position title, and contact information here). 


\section{Active Surveillance Tool}

\section{What is it?}

Active surveillance is closely monitoring a prostate cancer patient's condition without giving any treatment until cancer progression is demonstrated. This would include a PSA doubling time that occurs in $<3$ years or a tumor grade progression to Gleason score $\geq 7$. This approach is commonly used in men with early stage disease.

\section{Who is Appropriate for it?}

- Prostate-specific antigen (PSA) $\leq 10$

- Gleason score $\leq 6$ (tumor grade)

- T1c to T2a (tumor stage)

- For men with $>15$ year life expectancy, $<3$ core biopsies involved, $<50 \%$ of any core biopsy

\section{Follow-up Schedule}

- PSA, digital rectal exam (DRE) every 3 months for 2 years, then every 6 months thereafter (as long as PSA is stable)

- 10-12 core biopsies at 1 year, and then every 3 years until 80 years

- Optional transrectal ultrasound (TRUS) on alternate visits

\section{Intervention}

- For PSA doubling time $<3$ years

- For tumor grade progression to Gleason score $\geq 7$

PSA-based prostate cancer screening results in the diagnosis of prostate cancer in many men who will not have disease progression during their lifetime. Good-risk prostate cancer is defined as the following: a Gleason Tumor Grade of 6 or less, PSA < 10, and tumor staging T1c to T2a. In most of these cases, the disease is slow growing. It is estimated that the majority of newly-diagnosed men are not going to die as a result of their prostate cancer. Active surveillance may represent an appropriate strategy for some of these men.

A selective approach to treatment of favorable-risk prostate cancer is critical. It will provide a solution to the serious problem of over diagnosis, which can threaten the value of early detection of prostate cancer using PSA screening.

In order for this approach to be successful, the patient needs to understand and comply with the follow-up schedule. Without this cooperation, active surveillance will not achieve its intended goals. That is, almost all men undergoing active surveillance will die as a result of causes unrelated to prostate cancer.

I understand that my Active Surveillance follow-up care will be with Dr.

I have read, or have had read to me, the above information before signing this consent form. I agree to participate in Active Surveillance for my prostate cancer. I also authorize my permission to use or disclose my personal health information for the purpose of this follow-up. I have been offered ample opportunity to ask questions and have received answers that fully satisfy those questions.

Signature of Patient or Authorized Representative

Printed Name of Patient or Authorized Representative

Signature of the Person Obtaining Consent

Signature of Witness

\section{Date}

Date

\section{Date}

Date 24-hour Time

24-hour Time

A signed copy of this consent form must be given to each patient entering active surveillance for prostate cancer. 


\section{Prostate and GU Clinical Patient Navigation Program Patient Satisfaction Survey}

Please take a moment to share your experience with us. Your comments will be used to evaluate and improve our Prostate and Genitourinary Patient Navigator Program. Please mark only one answer for each question and return it in the postage-paid envelope provided.

Please circle the appropriate choice, with 1 being "poor" and 5 being "excellent."

(Poor)

The patient navigator was courteous.

The patient navigator was friendly.

The patient navigator was sensitive.

The patient navigator was respectful.

The patient navigator was thorough.

The educational materials I received were helpful, clearly stated, and easily understood.

Support services referrals met my needs.

I would recommend this service to others.

$\begin{array}{ll}1 & 2 \\ 1 & 2 \\ 1 & 2 \\ 1 & 2 \\ 1 & 2 \\ 1 & 2\end{array}$

2
2
2
2
2
2

3

45

345

$3 \quad 4 \quad 5$

345

$3 \quad 4 \quad 5$

3

4

5

$\begin{array}{lllll}1 & 2 & 3 & 4 & 5 \\ 1 & 2 & 3 & 4 & 5\end{array}$

Comments:

I utilized the services of the Patient Navigator $(\checkmark)$ Yes $\square$ No $\square$ If NO, why?

Do you have suggestions for improving this service?

If you would like to discuss this survey or your experience further, please include your name and contact information.

Name:

Best time to contact you:

Telephone (or other contact information): 


\section{Figure 2. Prostate Gancer Clinic Quality Indicators}

\begin{tabular}{|c|c|c|}
\hline Indicator & Rationale & Definition \\
\hline Volume & $\begin{array}{l}\text { Measure impact of CSSP (Clinical } \\
\text { Service Strategic Plan) initiatives on } \\
\text { patient and procedure volumes }\end{array}$ & Maine Medical Center Prostate Clinic \\
\hline & & Patient navigator utilization \\
\hline Screening/Enrollment in Clinical Trials & $\begin{array}{l}\text { Measuring access to advanced } \\
\text { treatment options }\end{array}$ & $\begin{array}{l}\text { Percentage of patients enrolled in } \\
\text { clinical trials } \\
\text { Percentage of patients screened for } \\
\text { clinical trials }\end{array}$ \\
\hline Access to Support Services & Ensuring completeness of care & $\begin{array}{l}\text { Percentage of patients accessing } \\
\text { nutritional and psychosocial support }\end{array}$ \\
\hline Timeliness of Treatment & Ensuring timeliness of care & Time from presentation to treatment \\
\hline $\begin{array}{l}\text { Multidisciplinary Conference } \\
\text { Attendance }\end{array}$ & $\begin{array}{l}\text { CoC (Commission on Cancer) } \\
\text { requires the Oncology Steering } \\
\text { Committee to set, monitor, and track } \\
\text { physician attendance }\end{array}$ & $\begin{array}{l}\text { Urology Conference requires surgery, } \\
\text { pathology, radiology, radiation } \\
\text { oncology, and medical oncology } \\
\text { participation }\end{array}$ \\
\hline Informed Decision Making & $\begin{array}{l}\text { Ensuring patient is fully informed of } \\
\text { treatment options }\end{array}$ & $\begin{array}{l}\text { Percentage of patients offered } \\
\text { a consult with a urologist and a } \\
\text { radiation oncologist }\end{array}$ \\
\hline Surgical Margin Positivity Rate & $\begin{array}{l}\text { Leading indicator for surgical } \\
\text { outcomes }\end{array}$ & $\begin{array}{l}\text { Percentage of prostatectomy patients } \\
\text { with positive margins }\end{array}$ \\
\hline Radiation Therapy: Brachytherapy & $\begin{array}{l}\text { Relapse free survival is greater for } \\
\text { patients receiving D90>130Gy vs. } \\
\text { patients receiving D90 <130Gy } \\
\qquad \vee 100\end{array}$ & Post-implant dosimetry \\
\hline Radiation Therapy: 3D Radiotherapy & $\begin{array}{l}\text { 3D conformal radiation therapy (CRT) } \\
\text { reduces both acute and normal tissue } \\
\text { toxicity in patient with prostate cancer } \\
\text { and allows higher cumulative doses to } \\
\text { be delivered }\end{array}$ & $\begin{array}{l}\text { Percentage of patients receiving } \\
\text { external beam radiation therapy } \\
\text { (EBRT) to the prostate only (no } \\
\text { metastases) who receive 3D radiation } \\
\text { therapy or IMRT }\end{array}$ \\
\hline Bladder Neck Contracture & $\begin{array}{l}\text { Monitoring acute complications to } \\
\text { treatment }\end{array}$ & $\begin{array}{l}\text { After surgery or radiation therapy, } \\
\text { treatment for bladder neck or urethral } \\
\text { stricture }\end{array}$ \\
\hline $\begin{array}{l}\text { Patient Assessment of Quality of Life } \\
\text { (QOL) Indicators }\end{array}$ & $\begin{array}{l}\text { Leading indicator for treatment } \\
\text { outcomes }\end{array}$ & $\begin{array}{l}\text { Percentage of patients assessed for } \\
\text { urinary, sexual, and bowel function } \\
\text { post-treatment }\end{array}$ \\
\hline
\end{tabular}

